\title{
Nanoparticle-impact experiments are highly sensitive to the presence of adsorbed species on electrode surfaces
}

\author{
Enno Kätelhön*Wei Cheng*Christopher Batchelor-McAuley* \\ Kristina Tschulik*and Richard G. Compton*†
}

March 20, 2014

\begin{abstract}
We theoretically and experimentally investigate the influence of partial surface blocking on the electrochemistry of nanoparticles impacting at an electrode. To this end, we introduce an analytical model for the adsorption of single blocking molecules on the electrode and calculate the resulting fractional electrode coverage. We find that even small amounts of adsorbed molecules can fully suppress detection of impacts of nanoparticles while the electrode characteristics in the detection of electroactive molecules hardly change. Our findings are supported by experimental data on the indigo nanoparticle electro-reduction at a carbon microelectrode (5.5 $\mu \mathrm{m})$ in aqueous solution. We find that nanoimpacts are fully suppressed in the presence of acetone at concentrations of $250 \mathrm{nM}$ which have a negligible effect on the electrode kinetics of the $\mathrm{Fe}(\mathrm{CN})_{6}^{3-/ 4-}$ couple.
\end{abstract}

\section{Introduction}

Due to a rapidly growing interest in the detection and characterisation of nanoparticles, research on nanoimpacts received increasing attention during the last years and was addressed in a variety of recent studies. While classical electrochemical measurements focus on the electrochemistry of analyte molecules in solution, nanoimpact experiments aim for the understanding of electrochemical and physical processes during the impact of nanoparticles on an electrode. ${ }^{1}$ Herein, processes can either be dominated by nanoparticle's intrinsic electrochemical properties ${ }^{2-4}$ or by reactions that are mediated through the nanoparticle during the time of its impact on the electrode surface under potential

\footnotetext{
*Physical and Theoretical Chemistry Laboratory, South Parks Road, Oxford, OX1 3QZ, England / United Kingdom.

${ }^{\dagger}$ Corresponding author. $\quad$ Tel: $\quad+44(0) \quad 1865 \quad 275957 . \quad$ E-mail: richard.compton@chem.ox.ac.uk
} 
control. ${ }^{5-11}$ This research enables new methods for the detection and characterization of nanoparticles and may enable the development of novel cost-efficient and portable sensors.

Even though the successful utilisation of nanoimpacts in sensing applications has been proven in a variety of different studies, electrode adsorption of trace organic material may remain a challenge in real samples. While the impact of this effect on the electrochemistry of electroactive molecules has been widely covered by previous research, ${ }^{12-18}$ no studies on the impact of partial electrode blocking on nanoimpact experiments have yet been published.

In this work, we present an analytical model for the adsorption of trace molecules present in solution on the electrode surface and compare the resulting fractional coverage during the detection of electroactive molecules and nanoparticles. We predict that even small amounts of adsorbing molecules, such as to give much less than monolayer coverage of adsorbate, can fully suppress the charge transfer between nanoparticles and the electrode, whereas the kinetics of molecular redox couples are negligibly influenced. We believe that this effect may often influence experimental results, since even ultra-pure water, which is frequently used in the preparation of sample solutions, may contain a few ppb of total organic carbon (TOC). ${ }^{19}$ Since the contaminating species are usually not further specified and may vary with the water used as raw material in the purification process, we here choose acetone as one example for a surface-active organic compound in the experiments supporting our analytical results: The trend seen in the analytical model is experimentally supported by data that demonstrates the suppression of impacts of indigo nanoparticles on a carbon electrode in presence of acetone in aqueous solution. We further speculate that the described effect may also may explain the lack of redox activity seen from large biomolecules when their electrochemical reaction is not mediated.

\section{Theory}

In this section, we introduce the theoretical model that is employed throughout the manuscript. We first illustrate the underlying assumptions of our approach before we derive an expression for the fractional surface coverage as a function of time and set it in relation to the effective fractional coverage that is observed in nanoimpact experiments.

\subsection{Theoretical model}

We investigate the time-dependent blocking of a macro electrode that is caused by the adsorption of trace molecules present in solution. We assume that the adsorbing molecules are initially equally distributed in solution and adsorb immediately and irreversibly whenever they touch a potentiostatted electrode. Once adsorbed, each molecule occupies a cylindrical volume on the electrode sur- 


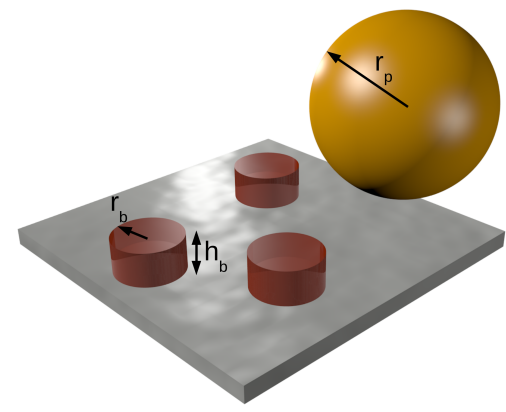

Figure 1: Graphical illustration of the employed theoretical model for nanoimpacts at a partially blocked electrode. Adsorbed molecules are modelled through cylindrical areas that cannot be accessed by nanoparticles.

face featuring the radius $r_{b}$ and the height $h_{b}$, while electron tunneling is fully suppressed within this volume so that the adsorption serves to inhibit electron transfer between the electrode and solution phase species. Such electrodes are referred to as 'partially blocked'. ${ }^{12}$

Spherical nanoparticles can react with the electrode surface at which they are oxidised or reduced and dissolve. Nanoparticles further feature a radius $r_{p}$ and cannot enter an area that is blocked by an adsorbed molecule. Thus, the effective electrode surface area available for nanoimpacts differs from the electroactive surface area, which is exposed to solution. A graphical illustration of the model can be found in Figure 1.

\subsection{Time-dependent fractional surface coverage}

In this section, we investigate the time-dependency of the fractional coverage $\theta$, which describes the fraction of the electrode surface area $A_{0}$ that is covered by adsorbing molecules and, thus, may take values between 0 and 1 . The surface area $A(t)$ of the electrode that is exposed to solution can therefore be calculated to the following expression at any point in time.

$$
A(t)=(1-\theta) A_{0}
$$

where $\theta=\theta(t)$ is a time-dependent function. The time dependency of $\theta$ hereby directly depends on the number of molecules $n_{a d s}(t)$ that are adsorbed at a certain time. Considering the area $A_{b}^{m}$ that every adsorbed molecule occupies on the surface, $A_{b}^{m}=\pi r_{b}^{2}, \theta(t)$ can be expressed through

$$
\theta(t)=\frac{A_{b}(t)}{A_{0}}=\frac{n_{a d s}(t) A_{b}^{m}}{A_{0}}=\frac{n_{a d s}(t) \pi r_{b}^{2}}{A_{0}}
$$

where $A_{b}$ represents the overall blocked surface area of electrode. 
In order to find an expression for $n_{a d s}(t)$, we now employ the Cottrell equation and modify it for the investigated system. In its original form, it provides an expression for the Faradaic current $i(t)$ of a macro electrode in the diffusionlimited case

$$
i(t)=\frac{n F A_{0} c_{0} \sqrt{D}}{\sqrt{\pi t}}
$$

where $n$ represents the number of electrons transferred per molecule, $F$ the Faraday constant, $c_{0}$ the initial concentration of reacting molecules, and $D$ the molecule's diffusion coefficient. ${ }^{20}$ However, if we now devide $i(t)$ by the charge $n e_{0}$ that is transferred during the reaction of a single molecule, we can now modify this expression to calculate the rate of adsorbing molecules. Furthermore, we substitute the electrode surface area $A_{0}$ by its exposed surface area $A(t)$, since the available surface area on the electrode decreases with time. While spherical diffusion to small electrode openings will dominate at large fractional coverages and the Cottrell equation is not applicable, this is nevertheless a good approximation for small values of $\theta$. Accordingly, we obtain

$$
\frac{d n_{a d s}}{d t}=\frac{i(t)}{n e_{0}}=\frac{N_{A} A(t) c_{0} \sqrt{D}}{\sqrt{\pi t}}
$$

where $N_{A}$ is the Avogadro constant. Using the expression for $A(t)$ in Equation (1), we can modify this result to

$$
\frac{d n_{a d s}}{d t}=\frac{N_{A} A_{0} c_{0} \sqrt{D}}{\sqrt{\pi}} \frac{(1-\theta)}{\sqrt{t}}
$$

If we now differentiate Equation (2) and substitute $d n_{a d s} / d t$, we obtain the following differential equation for the fractional coverage $\theta(t)$

$$
\frac{d \theta}{d t}=N_{A} r_{b}^{2} c_{0} \sqrt{\pi D} \frac{(1-\theta)}{\sqrt{t}}
$$

This equation is solved via separation of the variables $\theta$ and $t$

$$
\int d \theta \frac{1}{1-\theta}=N_{A} r_{b}^{2} c_{0} \sqrt{\pi D} \int d t \frac{1}{\sqrt{t}}
$$

and can be integrated to

$$
\ln (1-\theta)=N_{A} r_{b}^{2} c_{0} \sqrt{\pi D} \cdot 2 \sqrt{t}+\text { const } .
$$

Using the boundary condition $\theta(t=0)=0$, we then obtain an expression for the fractional coverage $\theta$ as a function of time

$$
\theta(t)=1-\exp \left(-2 N_{A} r_{b}^{2} c_{0} \sqrt{\pi D t}\right)
$$




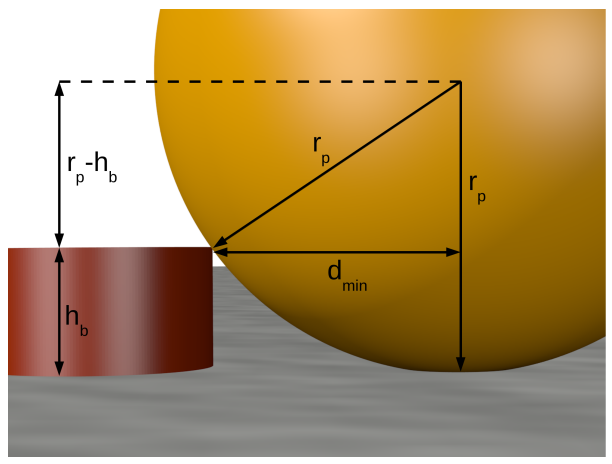

Figure 2: Schematic diagram for the calculation of the minimal distance $d_{\min }$ to a blocking molecule, at which a nanoparticle can touch the electrode surface.

\subsection{Effective fractional surface coverage in nanoimpact ex- periments}

Electroactive molecules that are dissolved in a sample typically feature a size of the same order of magnitude as the solvent molecules. Hence, the electrode surface area that is exposed to solution equals the surface area that is available for electrochemical reactions. However, this assumption does not hold if nanoparticles are used as analyte species. Since nanoparticles feature a distinct shape and a volume that is significantly larger than the volume of the solvent molecules, the surface area that is available for reaction may differ from the surface area that is exposed to solution. This effect can for instance be caused by electrode features such as a non-flat surface topology caused by adsorbed molecules.

In the following, we calculate the effective fractional coverage $\theta_{\text {eff }}$ for spherical nanoparticles that is observed in nanoimpact experiments as a function of the fractional coverage $\theta$ that can be found in experiments using small electroactive molecules. Herein, adsorbed molecules are again modelled through cylindrical volumes. We further assume that the effectively blocked areas $A_{b}^{n}$ do not overlap, which is a good approximation for a wide range of $\theta_{\text {eff }}$. For instance, in case of a hexagonal densest package of adsorbing molecules, this assumption holds up to a surface coverage of about $91 \%$. A schematic diagram of the model as well as variables used in the following calculations can be found in Figure 2.

As it can be seen in the diagram, the minimal distance $d_{m i n}$, at which a nanoparticle can touch the electrode surface, can be calculated through the Pythagorean theorem

$$
d_{\text {min }}=\sqrt{r_{p}^{2}-\left(r_{p}-h_{b}\right)^{2}}=\sqrt{2 r_{p} h_{b}-h_{b}^{2}}
$$

in case $h_{b}<r_{p}$. The electrode surface area that is blocked by a single adsorbing 
molecule is

$$
A_{b}^{m}=\pi r_{b}^{2}
$$

and

$$
A_{b}^{n}=\pi\left(r_{b}+d_{\text {min }}\right)^{2}
$$

in case of reacting electroactive molecules and nanoparticles, respectively. The effective surface coverage $\theta_{\text {eff }}$ can then be set in relation to the fractional coverage $\theta$ by comparison to the above calculated blocked surface areas $A_{b}^{m}$ and $A_{b}^{n}$.

$$
\frac{\theta_{\text {eff }}}{\theta}=\frac{A_{b}^{n}}{A_{b}^{m}}
$$

Hence, $\theta_{\text {eff }}$ can be expressed through

$$
\theta_{\text {eff }}=\theta \frac{\left(r_{b}+\sqrt{2 r_{p} h_{b}-h_{b}^{2}}\right)^{2}}{r_{b}^{2}}
$$

\section{Materials and methods}

Indigo (synthetic, 95\%) and dimethyl sulfoxide (DMSO, 99.9\%, anhydrous) were purchased from Aldrich. Indigo nanoparticles were synthesised using reprecipitation methods, as described previously. ${ }^{4}$ Briefly, Indigo was dissolved in DMSO to give $0.5 \mathrm{mM}$ indigo concentrations. $800 \mu \mathrm{L}$ of the indigo-DMSO solution was added to $20 \mathrm{~mL}$ of deionized water in a glass round-bottom flask and thoroughly mixed by magnetic vortex stirring at $800 \mathrm{rpm}$ for 5 minutes. The resulting mixture was a blue, transparent dispersion with the appearance of a solution. The size distributions of synthesised indigo nanoparticle were measured by dynamic light scattering (Malvern Instruments Ltd, UK). Electrochemical sizing of indigo nanoparticles was also carried out by analysis of the impact spikes observed using OriginPro 8.5.1 and SignalCounter developed by Dr. D. Omanović at the Center for Marine and Environmental Research Zagreb, Croatia.

Unless stated otherwise, all chemicals in this work were acquired from SigmaAldrich. All solutions were made up using ultrapure water of resistivity 18.2 $\Omega \mathrm{cm}$ (Millipore) at $25^{\circ} \mathrm{C}$ and degassed thoroughly with $\mathrm{N}_{2}$ (oxygen-free, BOC Gases plc) before use. The electrochemical experiments were performed at room temperature in a conventional three electrode setup using an Autolab II potentiostat (Metrohm-Autolab BV, Netherlands) within a double Faraday cage. For nano-impacting experiments and voltammetric measurements, a carbon microelectrode (radius $5.5 \mu \mathrm{m}$ ) was used as working electrodes, Saturated Calomel Electrode (SCE) as reference (Radiometer, Copenhagen), and a graphite rod as the counter electrode. 


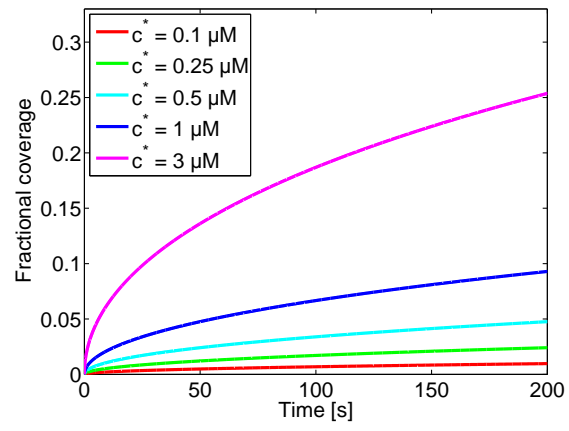

Figure 3: Fractional coverage as a function of time and $c^{*}$. Data was calculated for acetone in water and utilizes a diffusion coefficient $D$ of $1.16 \cdot 10^{-9} \mathrm{~m}^{2} \frac{1}{s}{ }^{21}$ and a cylinder radius $r_{b}$ of $r_{\text {acetone }}=0.308 \mathrm{~nm}$, which equals the molecular radius of acetone. ${ }^{22}$

\section{Results and discussion}

Figure 3 presents the fractional coverage of the electrode surface as a function of time. The graphs are calculated according to Equation (9) and utilize the diffusion coefficient of acetone as well as the radius of acetone molecules for the adsorbing molecules and the radius of the blocking cylinders, respectively. As it can be seen in the plot, the rate of adsorbing molecules significantly depends on their initial concentration in solution: at a concentration of $3 \mu \mathrm{M}$ the fractional coverage reaches a value of 0.05 within few seconds, while this value is not reached within the considered time interval of $200 \mathrm{~s}$ at lower concentrations. The fractional surface coverage is highly sensitive to the initial analyte concentration.

Figure 4 sets this result in relation to nanoimpact experiments. The dependency of the effective fractional coverage that is observed in the detection of nanoparticles is calculated according to the above derived Equation (14) and uses the parameters employed in Figure 3 as well as nanoparticles featuring the radius $r_{p}=46 \mathrm{~nm}$. Our calculations reveal a strong dependence of the effective fractional coverage $\theta_{\text {eff }}$ on the fractional coverage $\theta$ : in case of acetone molecules, a fractional coverage of only 0.1 percent, which hardly effects the electrode characteristics in the detection of electroactive molecules, effectively blocks more than 60 percent of the surface area in nanoimpact experiments. Many small blocked spots have a stronger effect on nanoimpacts than few larger ones at equal surface coverages. Considering the results in Figure 3, which show that small concentrations as low as $0.1 \mu \mathrm{M}$ cause blocking effects in conventional electrochemical experiments, we find that impact of adsorbing molecules on the detection of nanoparticles is significantly amplified. Hence, even small concentrations of adsorbing molecules may fully suppress reactions in nanoimpact experiments within few seconds as it can be seen in Figure 5, which demonstrates the effective fractional coverage as a function of time. 


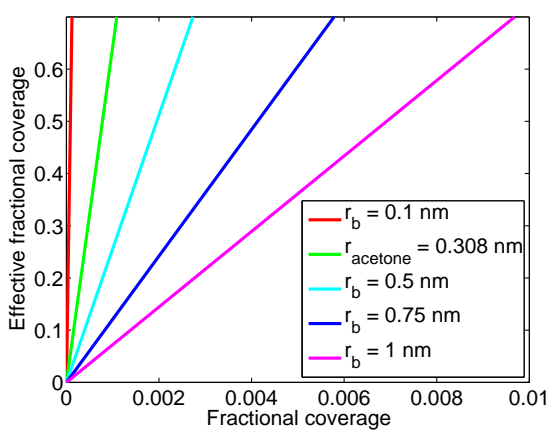

Figure 4: Effective fractional coverage $\theta_{\text {eff }}$ as a function of $\theta$ and the radius $r_{b}$ of the blocking cylinders. The calculations again use the diffusion coefficient of acetone. Furthermore, the height of the blocking cylinders is set to $2 r_{\text {acetone }}$ and the radius $r_{p}$ of the nanoparticles to $46 \mathrm{~nm}$.

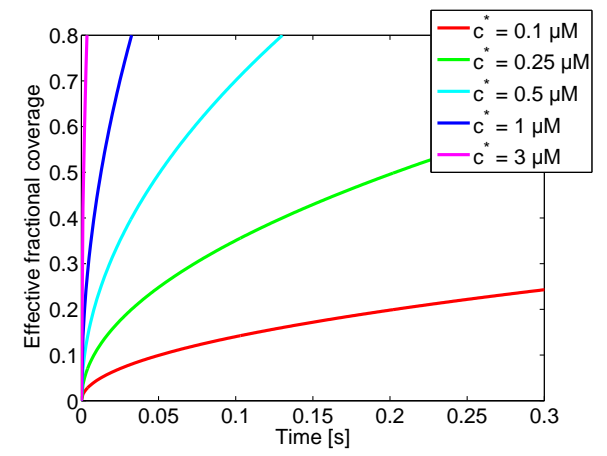

Figure 5: Effective fractional coverage as a function of time and $c^{*}$. Data was calculated for acetone in water and utilizes the parameter provided above.

Figure 6 presents experimental data of indigo $\left(\mathrm{C}_{16} \mathrm{H}_{10} \mathrm{~N}_{2} \mathrm{O}_{2}\right)$ nanoparticles impacting on a carbon microelectrode before and after the addition of acetone. During the experiment, a carbon microelectrode was placed in $137 \mathrm{mM} \mathrm{NaCl}$ solution and pre-dispersed indigo NPs were added. The electrode potential was held at a potential of $-700 \mathrm{mV}$, while reductive spikes from individual indigo NPs were observed under potentiostated conditions, see Figure 6a and 6c. As reported previously, ${ }^{4}$ those spikes result from the Faradaic reduction of indigo NPs at the electrode leading to dissolution of the nanoparticle in the form of the water soluble leuco-indigo. Afterwards, a known amount of 100 and $250 \mathrm{nM}$ acetone was added to the solution, which corresponds to about 3.6 and $9 \mathrm{ppb}$ of total organic carbon (TOC) and is close to the upper limit of TOC specified for common ultrapure water purification systems. ${ }^{19}$ After the addition of acetone, the number of reductive spikes was found to be significantly reduced 
(Figure 6b) and even completely suppressed at a higher concentration (Figure 6d), suggesting that the electrode may be partially or completely blocked for indigo nanoparticle impacts through adsorbing acetone molecules. ${ }^{23}$ However, the presence of the used amount of acetone hardly impacts the electrode characteristics in the detection of the molecular redox probe hexacyanoferrate: Cyclic voltammetry was performed using a carbon microelectrode in $8 \mathrm{mM} \mathrm{K}{ }_{3} \mathrm{Fe}(\mathrm{CN})_{6}$ and $137 \mathrm{mM} \mathrm{NaCl}$. Here we found that the voltammograms before and after adding acetone indicate that the electrodes surface area exposed to electroactive molecules has not significantly changed, which can be seen in the equal limiting currents before and after the addition of acetone, see Figure 7. Nonetheless, the voltammograms exhibit a small difference in the region near $+100 \mathrm{mV}$ vs SCE, where we obtain a slight decrease in reductive current during the exposure to acetone. We presume that this effect is caused by a change in the apparent standard rate constant $k_{0}$ and results from small amounts of acetone adsorbing on the microelectrode. Yet we refrain from a detailed kinetic analysis at this point, since the system is close to the electrochemically reversible limit in both cases and the determination of $k_{0}$ through the presented data is hence not sufficiently reliable.

The theoretical model used in Figure 3 and 5 is based on the Cottrell equation and, hence, models the case of linear diffusion, which does not apply in case of microelectrodes on the here considered time scale (see Section 2.2). However, the calculations that relate the fractional coverage $\theta$ to the effective fractional coverage $\theta_{\text {eff }}$ in Section 2.3 are independent from the electrode size. Therefore, we find a similar relation between $\theta$ and $\theta_{\text {eff }}$ in the theoretical model and in the presented experimental data: the experimental result supports the analytically found trend, which predicts a significantly stronger impact of adsorbing molecules on nanoimpact experiments in comparison to the detection of electroactive molecules.

\section{Conclusions}

In this study, we investigate the impact of partial electrode blocking on nanoimpact experiments. To this end, we develop an analytical model for the fractional electrode coverage as a function of time and derive an expression that relates the fractional coverage in nanoimpact experiments to the fractional coverage in the detection of electroactive molecules. Hereby, we find that even small amounts of adsorbing molecules can fully suppress nanoimpacts, while the electrode characteristics negligibly change in conventional electrochemical measurements on molecular targets. This is illustrated by experimental data showing the electrode blocking through acetone molecules during the detection of indigo nanoparticles whilst under exactly the same conditions a negligible effect on molecular electrochemistry is seen.

We further speculate that partial electrode blocking through contaminating molecules may explain observations made in electrochemical experiments involving large biomolecules. Here, electron transfer 

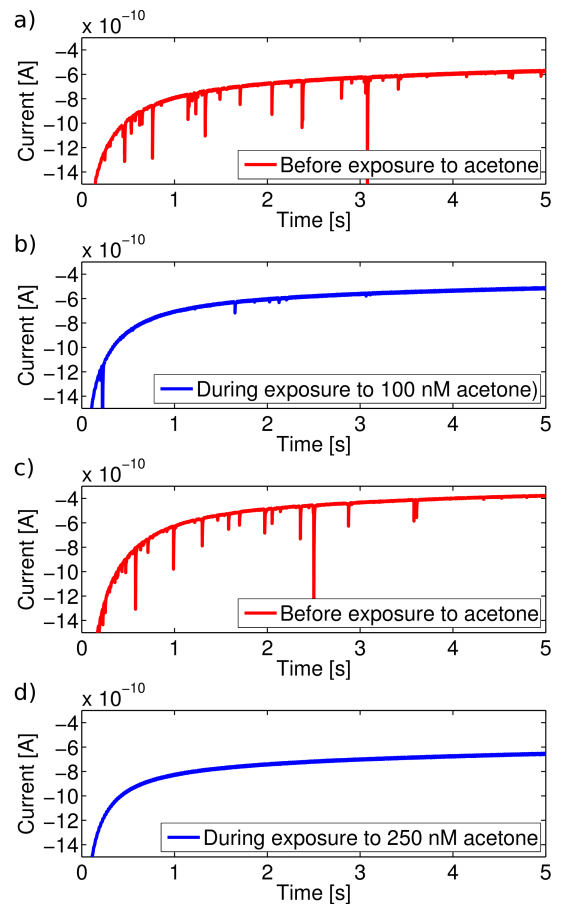

Figure 6: Indigo nanoparticles impacting at a carbon microelectrode. Data was recorded before, (a) and (c), and after the addition of 100 and $250 \mathrm{nM}$ acetone to the bulk solution, (b) and (d). The electrode was potentiostatted to +100 $\mathrm{mV}$ vs SCE.

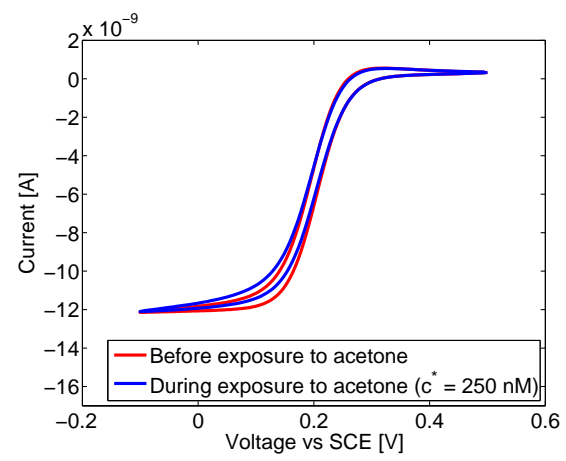

Figure 7: Cyclic voltammograms using a carbon microelectrode in a $8 \mathrm{mM}$ hexacyanoferrate solution before and during the exposure to $250 \mathrm{nM}$ acetone.

to or from electrodes is usually realised via either electron transfer mediation or fully irreversible adsorption of the biomolecule itself, while a direct reversible reaction can typically not be observed. This 
fact may be explained at least in part by adsorbing molecules that hinder the biomolecules from entering the region of electron transfer.

\section{Acknowledgments}

The research leading to these results has received partial funding from the European Research Council under the European Union's Seventh Framework Programme (FP/2007-2013) / ERC Grand Agreement n. [320403]. Wei Cheng gratefully acknowledges funding by the Leverhulme Trust. This research was further supported by a Marie Curie Intra European Fellowship within the 7th European Community Framework Programme (Kristina Tschulik).

\section{References}

[1] K. Tschulik, C. Batchelor-McAuley, H.-S. Toh, E. J. E. Stuart, and R. G. Compton. Electrochemical studies of silver nanoparticles: a guide for experimentalists and a perspective. Phys. Chem. Chem. Phys., 16:616-623, 2013.

[2] Y.-G. Zhou, N. V. Rees, and R. G. Compton. The electrochemical detection and characterization of silver nanoparticles in aqueous solution. Angew. Chem. Int. Ed., 50:4219-4221, 2011.

[3] E. J. E. Stuart, N. V. Rees, J. T. Cullen, and R. G. Compton. Direct electrochemical detection and sizing of silver nanoparticles in seawater media. Nanoscale, 5:174-177, 2012.

[4] W. Cheng, X.-F. Zhou, and R. G. Compton. Electrochemical sizing of organic nanoparticles. Angew. Chem. Int. Ed., 52:12980-12982, 2013.

[5] M. Heyrovský and J. Jirkovský. Polarography and voltammetry of ultrasmall colloids: Introduction to a new field. Langmuir, 11:4288-4292, 1995.

[6] M. Heyrovský, J. Jirkovský, and B. R. Müller. Polarography and voltammetry of aqueous colloidal $\mathrm{SnO}_{2}$ solutions. Langmuir, 11:4293-4299, 1995.

[7] M. Heyrovský, J. Jirkovský, and M. Štruplová Bartáčková. Polarography and voltammetry of aqueous colloidal $\mathrm{TiO}_{2}$ solutions. Langmuir, 11:43004308, 1995.

[8] X. Xiao and A. J. Bard. Observing single nanoparticle collisions at an ultramicroelectrode by electrocatalytic amplification. J. Am. Chem. Soc., 129:9610-9612, 2007.

[9] X. Xiao, F.-R. F. Fan, J. Zhou, and A. J. Bard. Current transients in single nanoparticle collision events. J. Am. Chem. Soc., 130:16669-16677, 2008. 
[10] N. V. Rees, Y.-G. Zhou, and R. G. Compton. Making contact: charge transfer during particle-electrode collisions. RSC Adv., 2:379-384, 2011.

[11] J. M. Kahk, N. V. Rees, J. Pillay, R. Tshikhudo, S. Vilakazi, and R. G. Compton. Electron transfer kinetics at single nanoparticles. Nano Today, 7:174-179, 2012.

[12] C. Amatore, J.M. Savéant, and D. Tessier. Charge transfer at partially blocked surfaces. a model for the case of microscopic active and inactive sites. J. Electroanal. Chem., 147:39-51, 1983.

[13] R. Baronas, F. Ivanauskas, and A. Survila. Simulation of electrochemical behavior of partially blocked electrodes under linear potential sweep conditions. J. Math. Chem., 27:267-278, 2000.

[14] F. G. Chevallier, T. J. Davies, O. V. Klymenko, L. Jiang, T. G. J. Jones, and R. G. Compton. Numerical simulation of partially blocked electrodes under cyclic voltammetry conditions: influence of the block unit geometry on the global electrochemical properties. J. Electroanal. Chem., 577:211$221,2005$.

[15] F. G. Chevallier, T. J. Davies, O. V. Klymenko, L. Jiang, T. G. J. Jones, and R. G. Compton. Influence of the block geometry on the voltammetric response of partially blocked electrodes: Application to interfacial liquidliquid kinetics of aqueous vitamin B12S with random arrays of femtolitre microdroplets of dibromocyclohexane. J. Electroanal. Chem., 580:265-274, 2005 .

[16] T. J. Davies, C. E. Banks, and R. G. Compton. Voltammetry at spatially heterogeneous electrodes. J. Solid State Electrochem., 9:797-808, 2005.

[17] F. G. Chevallier, N. Fietkau, J. del Campo, R. Mas, F. X. Muñoz, L. Jiang, T. G. J. Jones, and R. G. Compton. Experimental cyclic voltammetry at partially blocked electrodes: Elevated cylindrical blocks: Significantly blocked and non-flat electrodes can appear to show one-dimensional diffusion. J. Electroanal. Chem., 596:25-32, 2006.

[18] A. S. Barnes, N. Fietkau, F. G. Chevallier, J. Campo, R. Mas, F. X. Muñoz, T. G. J. Jones, and R. G. Compton. Electrochemical sizing of hemispherical microdroplets immobilized as regular arrays on partially blocked electrodes. J. Electroanal. Chem., 602:1-7, 2007.

[19] http://www.millipore.com, February 2014.

[20] R. G. Compton and C. E. Banks. Understanding Voltammetry, 2nd Edition. Imperial College Press, 2011.

[21] E. L. Cussler. Diffusion: Mass Transfer in Fluid Systems. Cambridge University Press, 1997. 
[22] A. B. Nadykto and F. Yu. Uptake of neutral polar vapor molecules by charged clusters particles: Enhancement due to dipole-charge interaction. J. Geophys. Res., 108:4717, 2003.

[23] C. Batchelor-McAuley, L. M. Gonçalves, L. Xiong, A.. Barros, and R. G. Compton. Controlling voltammetric responses by electrode modification; using adsorbed acetone to switch graphite surfaces between adsorptive and diffusive modes. Chem. Commun., 46:9037-9039, 2010.

\section{Keywords}

- Nanoelectrochemistry

- Nanoparticles

- Partially blocked electrodes

- Nanoimpacts

- Electrode adsorption 